\title{
Inventory Management of Railway Logistics Park Based on Artificial Neural Network
}

\author{
$\mathrm{Li} \mathrm{Gao}^{1 *}$, Huandi Dou ${ }^{2}$ \\ ${ }^{1}$ Xi'an Traffic Engineering Institute, Xi'an 710300, China \\ ${ }^{2}$ Transportation Operations monitoring Center of Shaanxi, Xi'an710075, China
}

Corresponding Author Email: gaoli006611@163.com

https://doi.org/10.18280/jesa.530514

Received: 1 June 2020

Accepted: 28 September 2020

\section{Keywords:}

artificial neural network (ANN), railway logistics park (RLP), inventory prediction, inventory management

\begin{abstract}
In recent years, China has stepped up its support to the optimization and development of railways. Meanwhile, the development of modern information technology (IT) has enhanced the economic advantages of railway logistics. To intelligently manage the inventory of railway logistics park (RLP), this paper integrates artificial neural network (ANN) into RLP inventory management. Firstly, the functional demand of RLP inventory management was analyzed comprehensively, and the main factors affecting the inventory demand were divided into different categories. Then, the authors formulated the framework of intelligent inventory management for RLP, and put forward the strategy of continuous periodic inventory monitoring. Finally, a RLP inventory prediction model was constructed based on optimized genetic algorithm (GA) and backpropagation neutral network (BPNN), and proved effective through experiments. The research results provide reference for the application of ANN in inventory management and prediction in other logistics fields.
\end{abstract}

\section{INTRODUCTION}

As the core logistics system in China, railway logistics gains advantage and realizes centralized transport from the numerous railway stations and lines across the country [1-3]. With the development of information technology (IT), the economic advantages of railway logistics have been highlighted in production and operation (P\&O), and infrastructure. By virtue of railway development and resource allocation, railway logistics can fully integrate various functions of modern logistics, such as transport, storage, containerization, and processing [4-8].

One of the important components of railway logistics park (RLP) is logistics park warehouse, which needs to optimize the flow of inventory goods in space and time [9-11]. In-depth research on the management strategy of warehouse inventory is necessary to make the RLP's functions more complete, services more flexible, and storage location and pickup stabler and safer.

Unlike other logistics parks, RLP mainly serves as the forwarding agent of railway transport alone. Therefore, RLP has certain limitations in the scope and coverage of business. In addition, the business efficiency of RLP is relatively low, owing to the late start of the construction of information network platform [12-16].

Kiesel et al. [17] examined the features of goods transport and storage of Qinghai Railway, and optimized RLP structure in terms of good diversity, marketing strategy, and intermodal transport, based on the production situation of railway goods and the design principles of RLP. From the perspectives of logistics safety, greenness, and social benefits, Binder et al. [18] identified the factors affecting RLP's operational benefits, constructed a reasonable evaluation index system (EIS), and provided the intelligent park management center with the top and bottom design techniques for operation management platform. Kumru and Kumru [19] planned and designed the technical and functional architectures for intelligent RLP inventory management, which involves the goods dispatching command center, goods sales and distribution center, comprehensive management center, and logistics information interaction center. Sharp et al. [20] provided new ideas for RLP intermodal transport and inventory management, with the aid of space-time positioning through crowd sensing, image recognition by multiple visual sensors, and adaptive selfoptimization by artificial intelligence (AI).

Some scholars have attempted to optimize the logistics path in logistics warehousing. Inspired by operations research, Lee et al. [21] optimized the inbound and outbound (I-O) paths of inventory goods through storage location transformation, and selected the most time-efficient path out of various logistics transport paths. Mohamed et al. [22] optimized the I-O sequences of inventory goods, and managed the supply chain system of logistics warehousing in a flexible manner. To formulate an ideal management strategy for inventory goods, and the scholars summarized the I-O paths of such goods, and achieved the minimal pickup and transport distance and maximal logistics efficiency by repositioning the storage locations.

Some scholars have discussed the arrangement of storage locations in logistics warehouse. Ha et al. [23] compared the strengths and merits of four optimization algorithms for storage locations in logistics parks, pointing out that the particle swarm optimization (PSO) has the best optimization effect. With the aim to minimize the logistics time, Nourbakhsh et al. [24] established a dynamic allocation strategy for storage locations in logistics parks, compared the effect of the strategy in different optimization algorithms (e.g. genetic algorithm (GA), fuzzy theory, and simulated annealing 
(SA) algorithm), and validated the strategy through example analysis.

Currently, RLP is faced with the following problems: the logistics equipment is not sufficiently intelligent, the management platform lacks top-level design, the data on the storage locations and transport states of goods are of poor quality, and the business scope and functions are not fully developed. To promote the multimodal transport and intelligent inventory management of RLP, this paper proposes a novel RLP inventory management strategy, based on the advantages of artificial neural network (ANN) in adaptability, self-learning, and self-optimization.

The remainder of this paper is organized as follows: Section 2 analyzes the functional demand of RLP inventory management, and divides the main factors affecting inventory demand into several categories; Section 3 formulates the framework of intelligent inventory management for RLP, and puts forward the strategy of continuous periodic inventory monitoring; Section 4 optimizes GA and backpropagation neutral network (BPNN), and constructs an inventory prediction model based on the optimized GA and BPNN; Section 5 verifies the effectiveness of the proposed prediction model through experiments; Section 6 puts forward the conclusions.

\section{DEMAND ANALYSIS}

Thanks to the rapid development of railway logistics, the industrial scale of RLP continues to expand with the increase in logistics amount. Meanwhile, the scope of RLP businesses, such as cost accounting and inventory management, also gradually widen. Figure 1 explains the workflow of RLP inventory management. Traditionally, RLP inventory management is completed manually. This traditional model has several problems: the workload of logistics tasks is gigantic, the cooperation between departments is inefficient, the storage locations are allocated unreasonably, the pickup paths are not reasonably planned, etc.

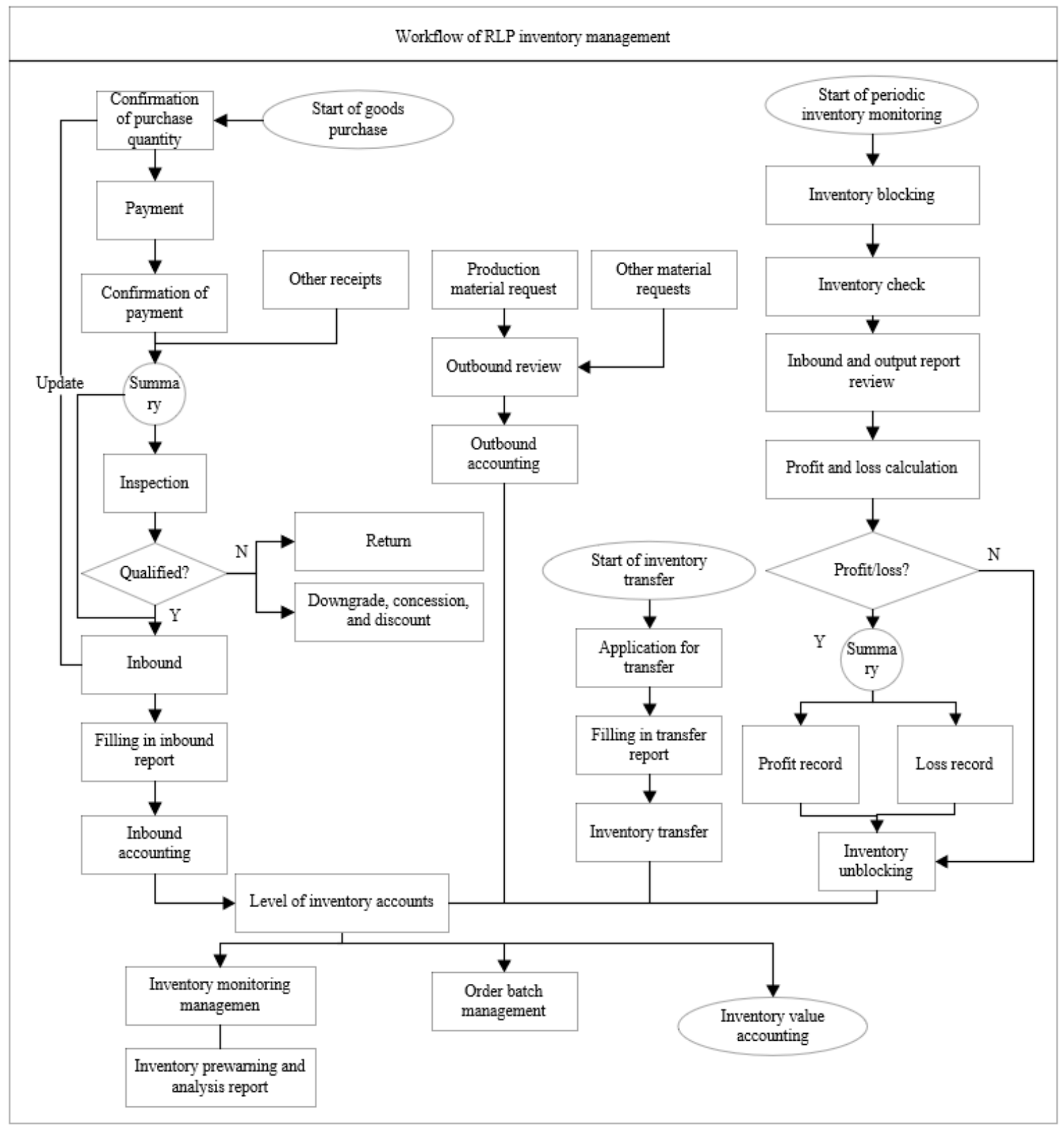

Figure 1. The workflow of RLP inventory management 
Considering the operation links and logistics flow of RLP, the inventory management demand of RLP was summed up in five aspects: basic information management, I-O management, user management, resource allocation management, and information query management.

Basic information management refers to the entry, modification, and maintenance of basic information on goods, warehouses, owners, and consumers, which are essential to the continuity of warehouse logistics operations.

I-O management mainly covers I-O review, I-O confirmation, and classification of I-O method. The information of inventory goods can be updated in real time, with the help of radiofrequency identification (RFID) labels.

User management focuses on the setting of owner and consumer rights. Resource allocation management stands for equipment call, material cleaning and scrapping, and inventory counting and allocation.

Information query management mainly refers to the query of physical operation log, total inventory, I-O reports, etc.

On this basis, a hierarchical framework of intelligent inventory management for RLP can be established as follows:

Layer 1 (goal):

$I=\{$ intelligent inventory management for RLP $\}$;

Layer 2 (main functions):
$I=\left\{I_{1}, I_{2}, I_{3}, I_{4}, I_{5}, I_{6}\right\}=\{$ basic information management, I-O management, user management, resource allocation management, information query management $\}$;

Layer 3 (specific functions):

$I_{1}=\left\{I_{11}, I_{12}, I_{13}, I_{14}\right\}=\{$ good information management, owner information management, warehouse information management, consumer information management $\}$;

$I_{2}=\left\{I_{21}, I_{22}, I_{23}\right\}=\{$ inbound review, inbound confirmation, classification of inbound method $\}$;

$I_{3}=\left\{I_{31}, I_{32}, I_{33}\right\}=\{$ outbound review, outbound confirmation, classification of outbound method $\}$;

$I_{4}=\left\{I_{41}, I_{42}\right\}=\{$ owner right setting, consumer right setting $\}$;

$I_{5}=\left\{I_{51}, I_{52}, I_{53}, I_{54}\right\}=$ request for equipment call, request for material cleaning and scrapping, estimation of good value, decision-making on cleaning and scrapping $\}$;

$I_{6}=\left\{I_{61}, I_{62}, I_{63}\right\}=\{$ query of physical operation log, query of total inventory, query of I-O report $\}$.

After setting up the hierarchical framework of intelligent inventory management, it is necessary to identify the reasons for the error in inventory demand prediction. The main influencing factors of RLP inventory demand were analyzed from two angles: the imbalance between railway logistics supply and market demand, and the three main players in RLP market, namely, supplier, RLP, and distributer. Figure 2 divides the main influencing factors into three categories.

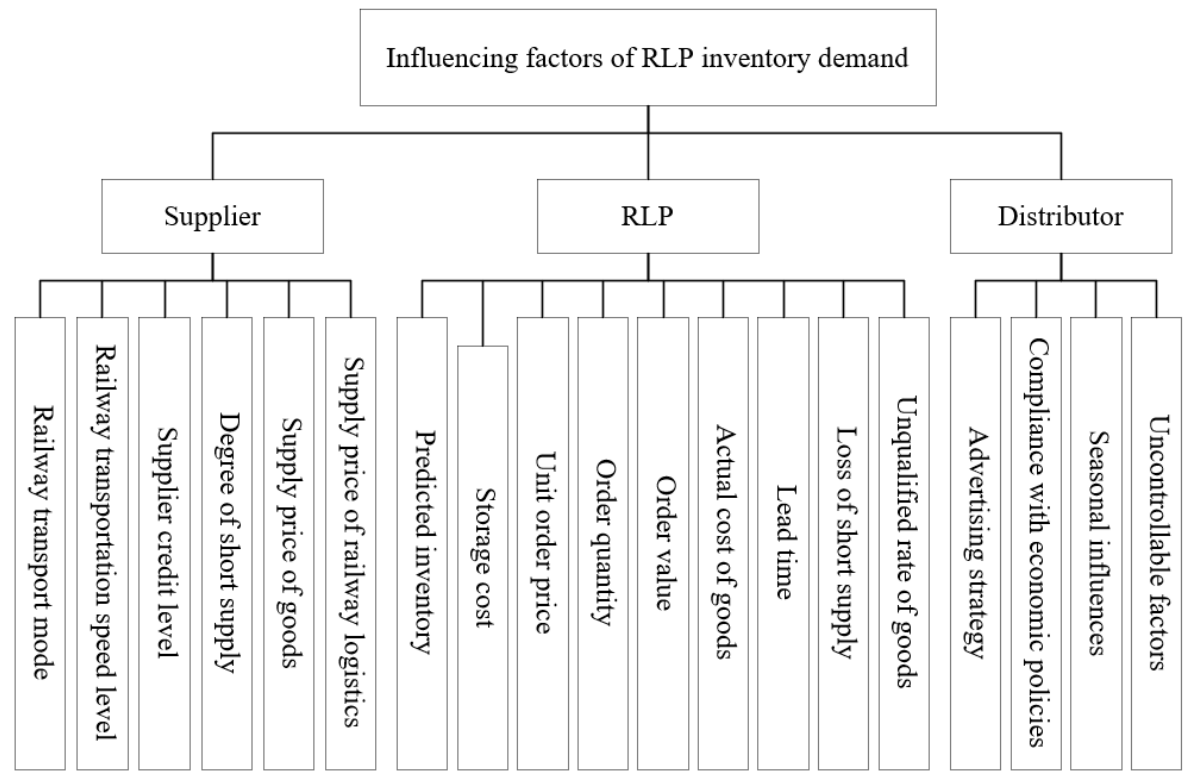

Figure 2. The division of the influencing factors

\section{CONTINUOUS PERIODIC MONITORING STRATEGY}

The existing studies on inventory monitoring mainly tackle supply-demand balance, the level of logistics supply, and inventory management strategies. From the perspective of good supply and demand, this paper designs an inventory calculation method that guarantees the continuity of RLP warehouse logistics. Figure 3 gives the block diagram of the proposed intelligent inventory management system for RLP.

During the replenishment, the good supply rate of RLP continues to change, making good demand highly uncertain. Let $M D_{a v}$ be the mean market demand per unit time, $M D_{S D}$ be the standard deviation of market demand, $T_{L}$ be the time difference between order placement to good delivery, and $\delta_{C}$ be ratio of time that inventory goods satisfy the demand of distributors to the total time of periodic replenishment. If the market demand for RLP goods per unit time obeys the normal distribution of $\left(M D_{a v}, M D_{S D}\right)$, then the market demand for RLP goods in period $T_{L}$ must satisfy the normal distribution of $\left(T_{L} M D_{a v}, T_{L} M D_{S D}\right)$. Hence, the inventory can be calculated by formula (1), during the continuous monitoring of RLP inventory.

$$
I N V=R T-T_{L} \cdot M D_{a v}
$$

where, $R T$ is the threshold for reordering. From order placement to the arrival of goods at RLP, the market demand can be expressed as $T_{L} M D_{a v}$. 


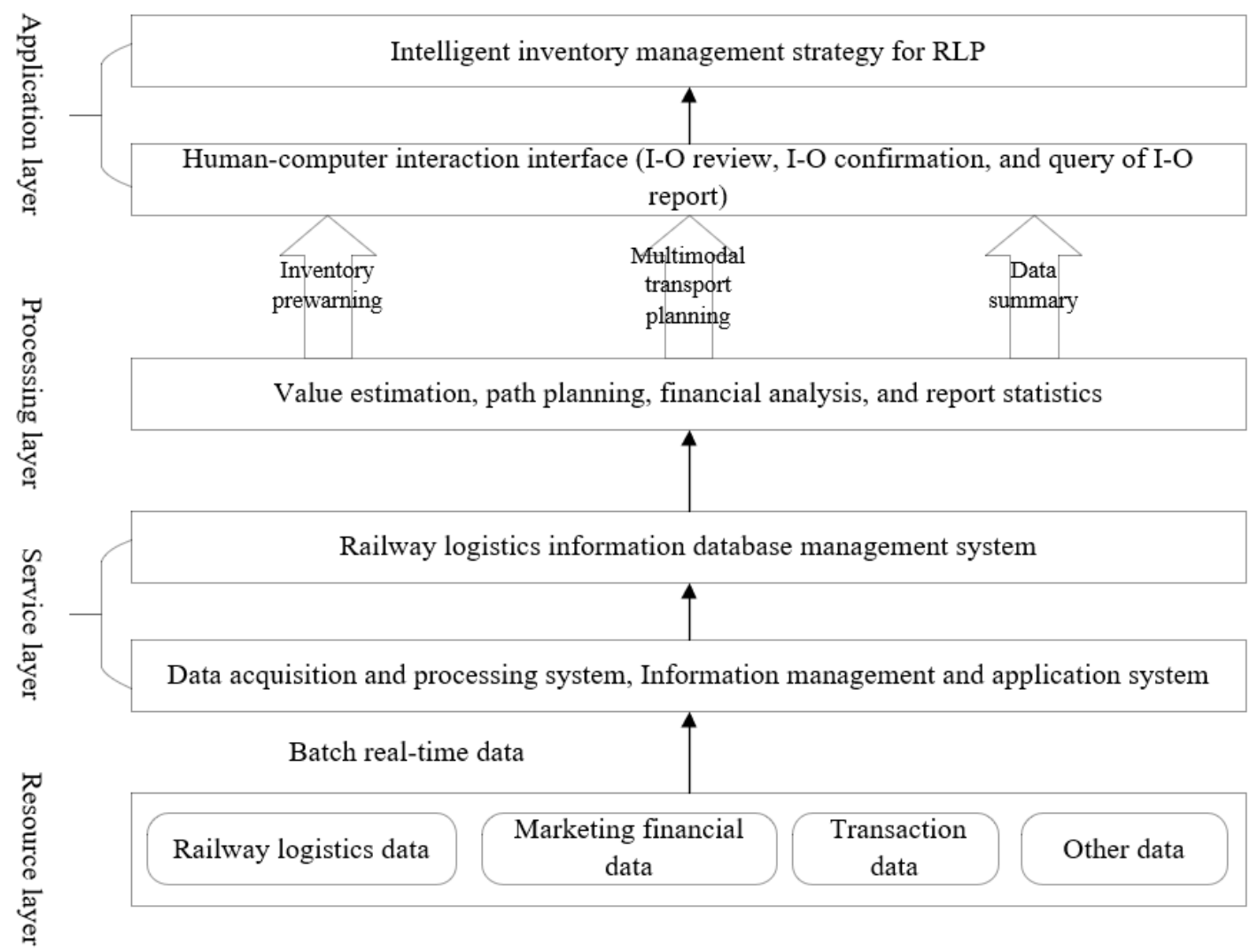

Figure 3. The block diagram of intelligent inventory management system for RLP

The inventory maximizes at the delivery of the ordered goods. Then, the standard normal distribution $F(*)$ can be defined as:

$$
F(a)=\int_{-\infty}^{a} \frac{1}{\sqrt{2 \pi} \sigma} a^{\frac{\sigma^{2}}{2}} d a
$$

Then, the periodic replenishment capacity of goods can be calculated by:

$$
\begin{aligned}
\delta_{C} & =F\left(\frac{R T-T_{L} \cdot M D_{a v}}{\sqrt{T_{L}} \cdot M D_{S D}}\right) \\
& =F\left(\frac{I N V}{\sqrt{T_{L}} \cdot M D_{S D}}\right)
\end{aligned}
$$

If the ratio $\delta_{C}$ is known, the inventory can be calculated by formula (3). Suppose the ratio of the demand satisfied by RLP inventory goods to the total demand in railway logistics market is $\delta_{S}$. The mean market demand $S S$ that cannot be satisfied before the delivery of the ordered goods can be calculated by:

$$
S S=O A \cdot\left(1-\delta_{S}\right)
$$

where, $O A$ is the order quantity. Then, we have:

$$
S S=\int_{R T}^{+\infty}(a-R T) f(a) d a
$$

Formula (5) can be simplified as:

$$
\begin{aligned}
S S & =-I N V\left[1-F\left(\frac{I N V}{\sqrt{T_{L}} \cdot M D_{S D}}\right)\right] \\
& =M D_{S D} f\left(\frac{I N V}{\sqrt{T_{L}} \cdot M D_{S D}}\right)
\end{aligned}
$$

where, $f(*)$ is the density function obeying standard normal distribution:

$$
f(a)=\frac{1}{\sqrt{2 \pi} \sigma} a^{-\frac{\sigma^{2}}{2}}
$$

If $S S$ or $\delta_{S}$ is known, the inventory $I N V$ can be solved by formulas (5) and (6).

In actual inventory management, the assumption of a fixed $T_{L}$, which is the precondition for analyzing the factors affecting inventory, does not hold. After all, the delivery of ordered goods is uncertain, owing to the delay in communication, changing weather, and uncertain transport conditions.

Let $T_{L-a v}$ be the mean time difference between order placement to delivery of goods, and $T_{L-S D}$ be the standard deviation of the time difference reflecting the uncertainty of supply. Considering the uncertainties in delivery and market demand, $T_{L-a v}$ and $T_{L-S D}$ were combined to predict and calculate the inventory.

The first step of periodical inventory monitoring is to configure the monitoring period $M P$. The maximum order quantity $O A_{\max }$ can be expressed as:

$$
O A_{\max }=\left(M P+T_{L}\right) M D_{a v}+I N V
$$


During the period $M P+T_{L}$, the mean demand of railway logistics market is $\left(M P+T_{L}\right) M D_{a v}$. Then, the periodic replenishment capacity of goods can be changed to:

$$
\begin{aligned}
\delta_{C}^{*} & =F\left(\frac{O A_{\max }-\left(M P+T_{L}\right) M D_{a v}}{\sqrt{M P+T_{L}} \cdot M D_{S D}}\right) \\
& =F\left(\frac{I N V}{\sqrt{M P+T_{L}} \cdot M D_{S D}}\right)
\end{aligned}
$$

If the ratio $\delta_{C}^{*}$ is known, the inventory can be calculated by formula (9).

However, the above inventory calculation method is not ideal, because the actual market demand for RLP goods does not necessarily obey normal distribution. The inventory reflects the changes of multiple factors, and implies the nonlinear relationship between railway logistics supply and market demand. From the angle of data mining, this paper decides to further analyze and predict RLP inventory with the help of ANN, which excels in the handling of nonlinear problems.

\section{INVENTORY PREDICTION MODEL}

\subsection{Optimization of GA}

Traditional GA has several disadvantages in the inventory optimization of logistics parks: low accuracy, large workload, and poor real-time monitoring effect. This paper replaces the binary encoding of traditional GA with floating-point encoding, achieving better optimization precision and operation efficiency. Figure 4 shows the workflow of the improved GA.

$$
h(x)=\left\{\begin{array}{c}
\left\|y_{\max }(x)-M[y(x)]\right\|+M[y(x)]-y(x) \\
0
\end{array}\right.
$$

where, $M[h(x)]$ is the mean fitness; $y_{m}(x)$ is the maximum of the input parameter in the current iteration:

$$
y_{\max }(x)=\max _{x \in X}\{y(x)\}
$$

The improved fitness function can change properly with the change of the calculation parameters, while remaining in the nonnegative state.

In addition, the crossover and mutation operators were improved by the Fibonacci method, which supports the onedimensional (1D) search for the minimum of the objective function along a fixed direction. The two operators were reconstructed under the quasi-optimal principle. Through crossover and mutation, each new individual can be expressed as:

$$
\left\{\begin{array}{l}
G_{i}^{*}=0.618 G_{i}+0.382 G_{j} \\
G_{j}^{*}=0.618 G_{j}+0.382 G_{i}
\end{array}\right.
$$

Let $g_{i j l}$ be the 1-th component of the j-th individual in the ith generation. Taking random numbers between 1 and 0 or within $\left[n_{l}, m_{l}\right]$, the mutation of the new individual can be

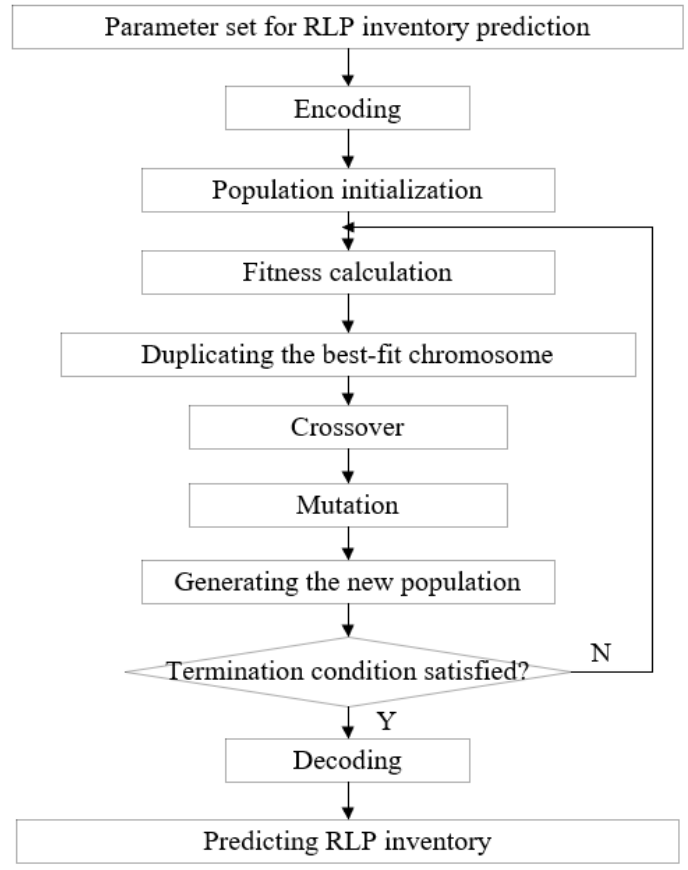

Figure 4. The workflow of the improved GA

The traditional fitness function can be expressed as:

$$
h(x)=\left\{\begin{array}{cc}
\rho_{\max }-y(x) & y(x)<\rho_{\max } \\
0 & \text { Otherwise }
\end{array}\right.
$$

To accurately predict the inventory, $h(x)$ should adapt to the changes in the calculation parameters of RLP inventory. To this end, formula (10) can be optimized into:

$y(x)<\left\|y_{\max }(x)-M[y(x)]\right\|+M[y(x)]$
Otherwise

expressed as:

$$
g_{(i+1) j l}=\left\{\begin{array}{l}
n_{l}+0.382\left(m_{l}-n_{l}\right) \quad \text { if } \operatorname{RAN}(0,1)=0 \\
m_{l}+0.618\left(m_{l}-n_{l}\right) \text { if } \operatorname{RAN}(0,1)=1
\end{array}\right.
$$

where, $R A N(*)$ is a random function. The GA performance can be improved substantially through the above steps.

\subsection{Optimization of BPNN}

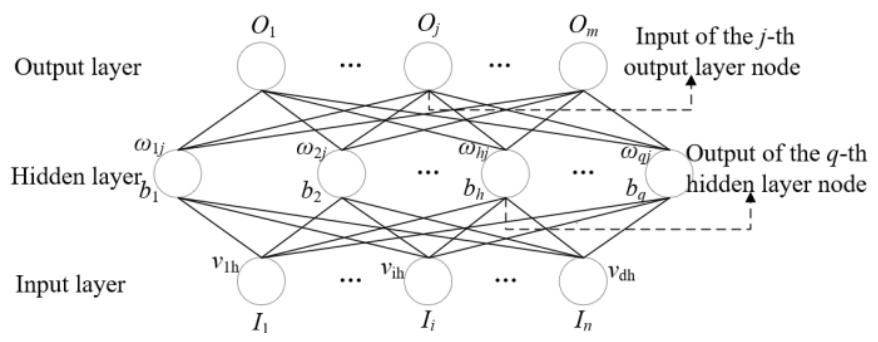

Figure 5. The structure of traditional BPNN

The structure of traditional BPNN is displayed in Figure 5. The traditional BPNN might fall into the local minimum or 
converge slowly in predicting the inventory of logistics parks. To solve the problem, BPNN was improved by introducing the momentum term, changing parameters, and adjusting the activation function.
Let $\omega(t)$ and $N G(t)$ be the connection weight and negative gradient of the network at time $t$, respectively. Then, the momentum term can be introduced by:

$$
\omega(t+1)=\omega(t)+\beta[(1-\lambda) N G(t)+\lambda N G(t-1)]=\omega(t)+\beta\left[(1-\lambda)\left(-\frac{\partial E F}{\partial \omega(t)}\right)+\lambda\left(-\frac{\partial E F}{\partial \omega(t-1)}\right)\right]
$$

where, $E F(*)$ is the error function.

During the prediction of RLP inventory, the network should avoid the local minimum by making use of the previous weight changes, i.e. the historical gradient direction, rather than focus on the parameter changes in the periods right before and after the current moment. Here, the network weight is adjusted through multi-step method:

$$
\Delta \omega_{j t}(k+1)=\lambda \alpha_{j} u_{i}+\beta\left[\rho_{s}^{0} \omega_{j i}(k)-\rho_{s}^{p} \omega_{j i}(k-1)+L+(-1)^{s} \rho_{s}^{p} \omega_{j i}(k-s)\right]=\lambda \alpha_{j} u_{i}+\beta \sum_{p=0}^{s}(-1)^{p} \rho_{s}^{p} \omega_{j i}(k-p)
$$

$$
\rho_{s}^{l}=\frac{s(s-1) P(s-p+1)}{s !}
$$

Following the multi-step method, the added momentum term fully considers the previously accumulated weight changes, and thus reduces the oscillation in learning and improves convergence. Besides, the randomly generated initial weight helps to avoid falling into local minimum.

The slow convergence of BPNN in RLP inventory prediction is attributable to the improper step length. If the step length is too small, the convergence will be too slow; if the step length is too long, the network tends to oscillate. Hence, the step length was adjusted by:

$$
L(t)=2^{\operatorname{sgn}[N G(t) N G(t-1)]} L(t-1)
$$

Formula (18) shows that the step length increases if its direction is the same as the gradient direction, and decreases if otherwise. In addition, the convergence speed of BPNN is also greatly affected by the learning rate $\lambda$. This influencing factor was optimized through adaptive adjustment:

$$
\lambda(t)=\left\{\begin{array}{cc}
1.06 \lambda(t-1) & E F(t)<E F(t-1) \\
0.85 \lambda(t-1) & E F(t)>1.05 E F(t-1) \\
\lambda(t-1) & \text { Otherwise }
\end{array}\right.
$$

Similarly, the step length was also optimized through adaptive adjustment:

$$
L=\left\{\begin{array}{cc}
0.96 & E F(t)<E F(t-1) \\
0 & E F(t)>1.05 E F(t-1) \\
L & \text { Otherwise }
\end{array}\right.
$$

To a certain extent, the activation function of BPNN determines the properties of $E F(*)$. Based on the slow converging sigmoid function, $E F(*)$ was analyzed as follows:

$$
\frac{\partial E F}{\partial \omega_{i j}}=-\left(O_{j}^{*}-O_{j}\right) O_{j}\left(1-O_{j}\right) I_{i}
$$

Formula (21) shows that, when the error of BPNN tends to the minimum, the inventory samples $O_{j}^{*}$ - $O_{j}$ approaches zero.
Therefore, the proneness of BPNN to local minimum is directly caused by the fact that $O_{j}$ or $1-O_{j}$ is approximately zero. Through the above analysis, the sigmoid function was improved as follows. First, the piecewise function was introduced:

$$
S(x)=\left\{\begin{array}{cc}
1 & \theta I>\pi / 2 \\
{[\sin (\theta I)+1] / 2} & |\theta I|<\pi / 2 \\
0 & \theta I<-\pi / 2
\end{array}\right.
$$

where, the $\theta$ value depends on learning rate $\lambda$ and step length $L$. Then, an adjustable bias $\mu$ was introduced to optimize the function into $S(I, r, \mu)=1 / 1+e^{-r(I+\mu)}$, which can move along horizontally with the setting of $\mu$. The improved piecewise function can greatly accelerate the convergence of BPNN.

The improved BPNN can be implemented in the following steps:

Step 1. Randomly initialize the connection weights and thresholds in $(0,1)$.

Step 2. Compute the calculation parameters for RLP inventory prediction, calculate the outputs of hidden layer and output layer nodes, and obtain the errors in network output $E F(t)$ and in the outputs of hidden layer and output layer nodes in this iteration.

Step 3. Optimize the momentum term, learning rate, and step length, and adjust the connection weights and thresholds.

Step 4. Import the samples of the calculation parameters for prediction RLP inventory in the next period to the network, return to Step 2 to execute iterative calculation until all samples are computed, terminate the network training, and obtain the maximum error $E F_{\max }(M)$ in the training process.

Step 5. Judge the maximum error $E F_{\max }(M)$ : If $E F_{\max }(M)$ is smaller than the preset error threshold, terminate the training; otherwise, go to Step 6.

Step 6. Adaptively adjust the optimal error threshold $v$ based on the mean difference between the maximum errors of the $\mathrm{M}$ th iteration and the M-1-th iteration:

$$
\Delta E F_{\text {max }}^{*}=\left[E F_{\text {max }}(M)-E F_{\text {max }}(M-2)\right] / 2
$$

If $\Delta E F^{*}{ }_{\text {max }}$ is greater than the control value determined to ensure convergence accuracy, then make $v=E F_{\max }(N) / 2$; otherwise, make $v=E F_{\max }(N)$.

Step 7. Select the samples of the calculation parameters for prediction RLP inventory from the training samples, import 
them to the network, and return to Step 2 to execute iterative calculation until $\Delta E F^{*}{ }_{\max }$ drops to $v$.

\section{EXPERIMENTS AND RESULT ANALYSIS}

Before verifying the effectiveness of our RLP inventory management strategy and model, the inventory of a RLP from January to December, 2019 were counted, and the current inventory cost of the RLP was analyzed. As shown in Table 1, the month-end inventory of the RLP maintained at about 32.26 million, more than 2 million above market demand. The unreasonably high inventory pushes up the inventory cost of the RLP, making economic benefits uncontrollable.

To lower inventory cost, realize effective inventory management, and make accurate inventory prediction, this paper sets up a BPNN for RLP inventory prediction. Table 2 provides the errors of the BPNN during the training on the samples of calculation parameters of the inventory. It can be seen that the BPNN performed fairly well in model training. The error reached 3.9\% in April, 2019, and 2.8\% in January of the same year, about $1 \%$ than the error in any other month. This means the stability of the BPNN can be further improved.

Table 1. The inventory of the RLP (unit: 10,000)

\begin{tabular}{cccc}
\hline Month & Inventory & Month & Inventory \\
\hline $2019-01$ & 3,267 & $2019-07$ & 3785 \\
$2019-02$ & 3,468 & $2019-08$ & 3117 \\
$2019-03$ & 3,175 & $2019-09$ & 2965 \\
$2019-04$ & 3,268 & $2019-10$ & 2983 \\
$2019-05$ & 3,319 & $2019-11$ & 3031 \\
$2019-06$ & 2,890 & $2019-12$ & 3452 \\
\hline
\end{tabular}

Table 2. The training errors of the BPNN on RLP inventory (unit: 10,000)

\begin{tabular}{ccccc}
\hline Month & True value & Fitted value & Relative error & Mean absolute Percentage error \\
\hline $2019-01$ & 2,785 & 2,777 & 0.002872531 & \\
$2019-02$ & 3,187 & 3,193 & -0.001882648 & \\
$2019-03$ & 3,421 & 3,416 & 0.001461561 & \\
$2019-04$ & 3,765 & 3,750 & 0.003984064 & \\
$2019-05$ & 3,298 & 3,301 & -0.000909642 & \\
$2019-06$ & 3,511 & 3,502 & 0.002563372 & \\
$2019-07$ & 3,763 & 3,772 & -0.002391709 & \\
$2019-08$ & 2,871 & 2,866 & 0.001741553 & \\
$2019-09$ & 2,963 & 2,971 & -0.002699966 & \\
$2019-10$ & 3,179 & 3,183 & -0.001258257 & \\
$2019-11$ & 3,479 & 3,477 & 0.000574878 & \\
$2019-12$ & 3,510 & 3,512 & -0.000569801 & \\
\hline
\end{tabular}

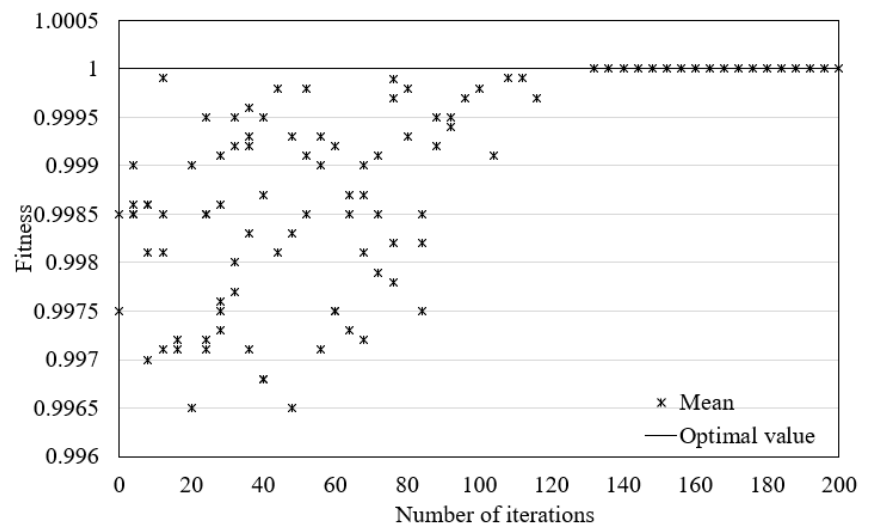

(a) Training

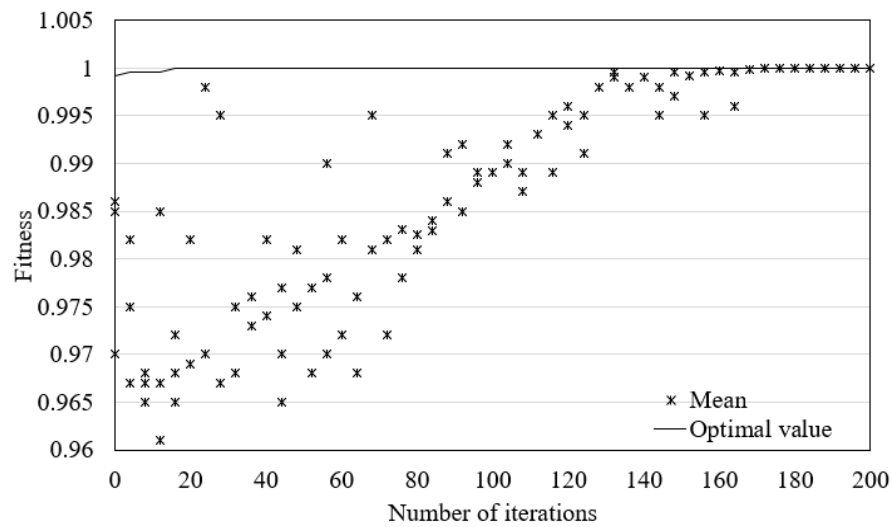

(b)Test

Figure 6. The evolution of the optimal GA

In this paper, the optimized GA performs optimized crossover and mutation on individuals. The values of crossover and mutation operators determine the algorithm performance in convergence and evolution. To improve the evolution and prediction results, the thresholds and weights corresponding to the best-fit individual were adopted as the optimal outputs of GA, and used to decode the optimal individual. Figure 6 presents the evolution processes of the optimal GA during the training and the test. It can be seen that the optimal GA started to converge at the 130-th iteration.

The optimal BPNN was trained with the weights and thresholds corresponding to the optimal individual, as outputted by the optimal GA. Figures 7 and 8 record the convergence curves and prediction effects of the optimal
BPNN in training and test, respectively. It can be seen that the optimal BPNN reached the preset objective error at the $50^{\text {th }}$ and $25^{\text {th }}$ iterations during the training and test on RLP inventory, respectively; the optimal BPNN achieved very small relative error and good fitting effect in both training and test, suggesting the effectiveness of the network in prediction.

For comparison, three RLP inventory prediction models were constructed based on traditional BPNN, fuzzy neural network (FNN), and PSO, respectively, and received the relevant training and test. The relative error and mean absolute percentage error of each model in training and test were calculated, and compared with those of our model (Table 3). It can be seen that our model predicted the RLP inventory accurately; the errors were slightly higher in March than those 
in other month, but lower than those of all the three contrastive models. In addition, our model had smaller difference between

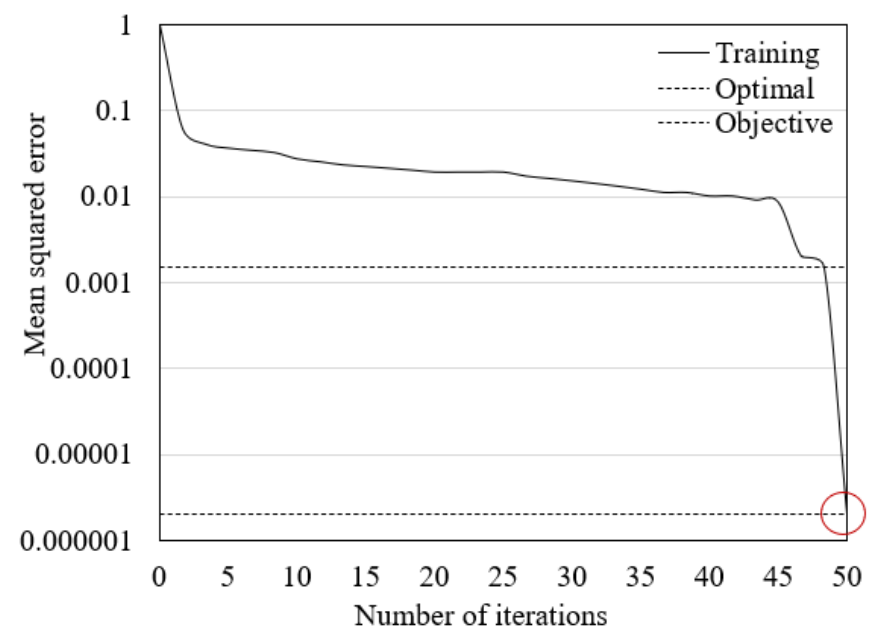

(a) Training monthly errors than any other model. These results fully demonstrate the stability of our prediction model.

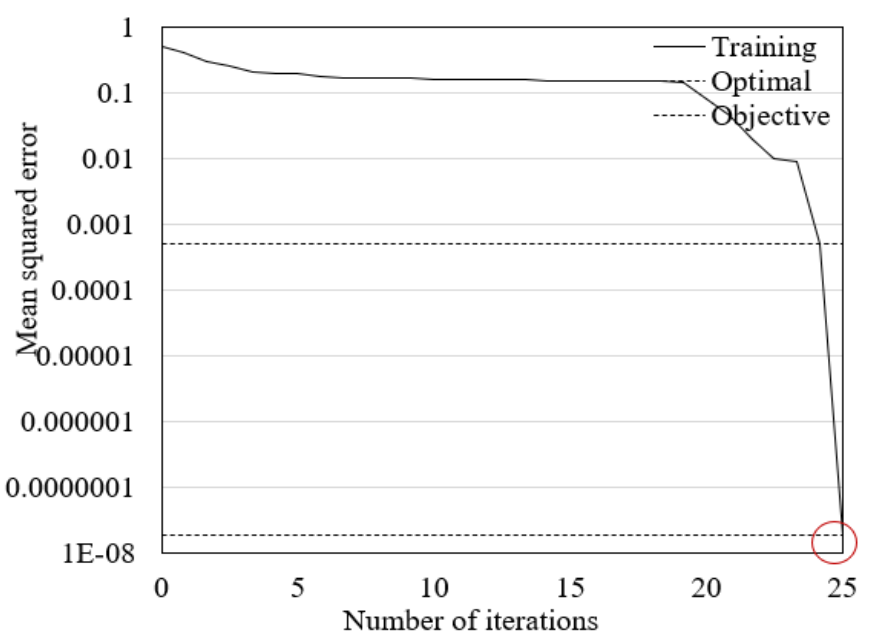

(b)Test

Figure 7. The convergence curves of the optimal BPNN

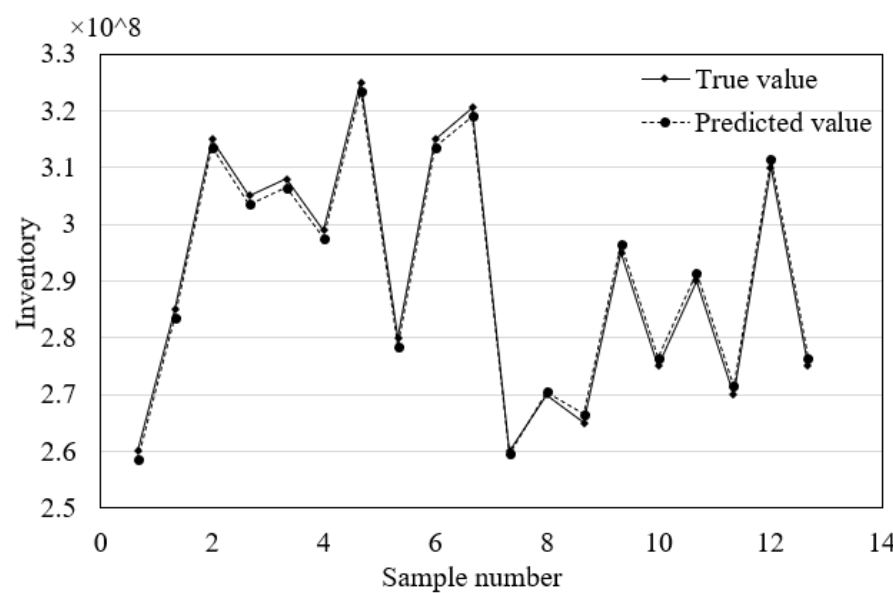

(a) Training

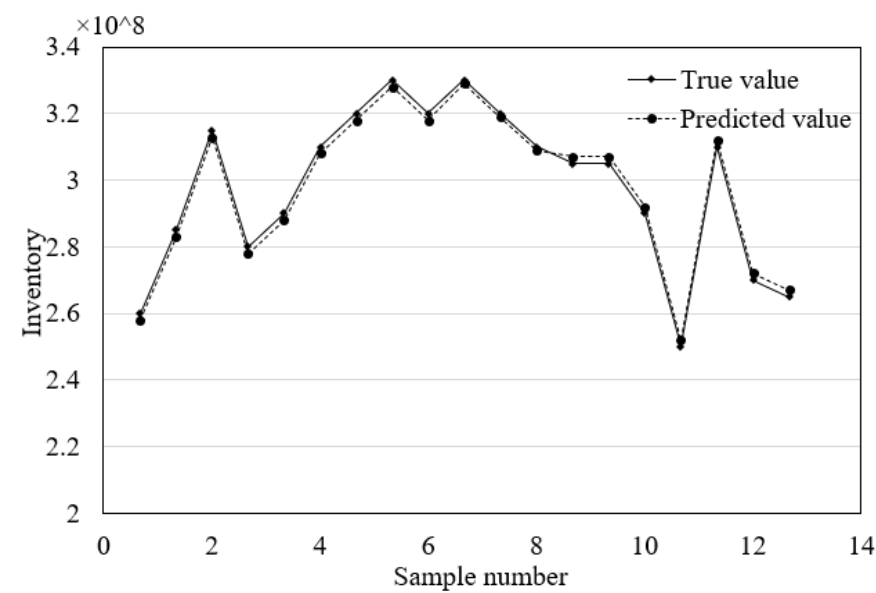

(b)Test

Figure 8. The prediction effects of the optimal BPNN

Table 3. The comparison between different prediction models (unit: 10,000)

\begin{tabular}{|c|c|c|c|c|c|c|}
\hline \multicolumn{2}{|c|}{ Model Month } & 2020.01 & 2020.02 & 2020.03 & 2020.04 & Mean absolute percentage error \\
\hline \multicolumn{2}{|c|}{ True value } & 3,124 & 2,954 & 2,994 & 3,256 & \multirow{3}{*}{$8.25 \%$} \\
\hline \multirow{2}{*}{ BPNN } & Predicted value & 3,413 & 2,778 & 2,758 & 3,116 & \\
\hline & Relative error & $9.25 \%$ & $9.34 \%$ & $7.88 \%$ & $7.37 \%$ & \\
\hline \multirow{2}{*}{ FNN } & Predicted value & 3299 & 3121 & 2775 & 3079 & \multirow{2}{*}{$6.01 \%$} \\
\hline & Relative error & $5.60 \%$ & $5.65 \%$ & $7.31 \%$ & $5.43 \%$ & \\
\hline \multirow{2}{*}{ PSO } & Predicted value & 3247 & 224 & 2904 & 3148 & \multirow{2}{*}{$3.78 \%$} \\
\hline & Relative error & $3.94 \%$ & $4.4 \%$ & $3.0 \%$ & $3.31 \%$ & \\
\hline & Predicted value & 3136 & 2937 & 2977 & 3225 & \multirow{2}{*}{$1.89 \%$} \\
\hline Our model & Relative error & $1.02 \%$ & $1.92 \%$ & $2.57 \%$ & $1.57 \%$ & \\
\hline
\end{tabular}

\section{CONCLUSIONS}

This paper fully explores the RLP inventory management based on ANN. Firstly, the functional demand of RLP inventory management was analyzed comprehensively, and the main factors affecting the inventory demand were divided into several categories. Next, a continuous periodic inventory monitoring strategy was designed under a self-designed framework for RLP intelligent inventory management system.
After that, the traditional GA and BPNN were optimized, and combined into a RLP inventory prediction model. Experimental results verify the good evolution and prediction effects of the optimized GA. In addition, the proposed prediction model was found to be more accurate and stable in RLP inventory prediction than traditional BPNN, FNN, and PSO. 


\section{REFERENCES}

[1] Kisielewski, P. (2016). The system of IT support for logistics in the rail transport. Archives of Transport, 40(4): 39-50. https://doi.org/10.5604/08669546.1225465

[2] Rouky, N., Couzon, P., Boukachour, J., Boudebous, D., Alaoui, A.E.H. (2018). Optimization of containers transfer in le Havre port: A new algorithm for the railway transportation system. IFAC-PapersOnLine, 51(11): 1676-1681. https://doi.org/10.1016/j.ifacol.2018.08.215

[3] Likitlersuang, S., Pholkainuwatra, P., Chompoorat, T., Keawsawasvong, S. (2018). Numerical modelling of railway embankments for high-speed train constructed on soft soil. Journal of GeoEngineering, 13(3): 149-159. http://dx.doi.org/10.6310/jog.201809 13(3).6

[4] Merlini, D., Stocker, D., Falanesca, M., Schuerch, R. (2018). The ceneri base tunnel: construction experience with the southern portion of the flat railway line crossing the Swiss Alps. Engineering, 4(2): 235-248. https://doi.org/10.1016/j.eng.2017.09.004

[5] Rengshausen, R., Weiner, T. (2018). Metro Doha Green Line-More than $30 \mathrm{~km}$ of tunnel in 18 months: Metro Doha Green Line-Mehr als $30 \mathrm{~km}$ Tunnel in 18 Monaten. Geomechanics and Tunnelling, 11(1): 50-61. https://doi.org/10.1002/geot.201700061

[6] Wanke, P., Kalam Azad, M.A. (2018). Efficiency in Asian railways: a comparison between data envelopment analysis approaches. Transportation Planning and Technology, 41(6): 573-599. https://doi.org/10.1080/03081060.2018.1488928

[7] Kozachenko, D., Vernigora, R., Kuznetsov, V., Lahvinova, N., Rustamov, R., Papahov, A. (2018). Resource-saving technologies of railway transportation of grain freights for export. Archives of Transport, 45(1): 53-64. https://doi.org/10.5604/01.3001.0012.0944

[8] Yoon, K., Gwak, D.Y., Chun, C., Seong, Y., Hong, J., Lee, S. (2018). Analysis of frequency dependence on short-term annoyance of conventional railway noise using sound quality metrics in a laboratory context. Applied Acoustics, 138: 121-132. https://doi.org/10.1016/j.apacoust.2018.03.024

[9] Okoh, P. (2019). Integrated logistics support and asset management (ILSAM). Infrastructure Asset Management, $6(4)$ : $245-257$ https://doi.org/10.1680/jinam.17.00026

[10] Sahin-Arslan, A., Ertem, M.A. (2019). A WAREHOUSE design with containers for humanitarian logistics: a reallife implementation from Turkey. International Journal of Industrial Engineering, 26(2): 139-155.

[11] Huang, W., Zhang, Y., Shuai, B., Xu, M., Xiao, W., Zhang, R., Xu, Y. (2019). China railway industry reform evolution approach: Based on the vertical separation model. Transportation Research Part A: Policy and Practice, 130: 546-556. https://doi.org/10.1016/j.tra.2019.09.049

[12] Consilvio, A., Di Febbraro, A., Meo, R., Sacco, N. (2019). Risk-based optimal scheduling of maintenance activities in a railway network. EURO Journal on
Transportation and Logistics, 8(5): 435-465. https://doi.org/10.1007/s13676-018-0117-z

[13] Noor, B.A., Sirong, Y., Alam Kazmi, S.H., Abid, M.M. (2016). Modeling for operating expenses and time consumption of high-speed railway (HSR). European Transport $\backslash$ Trasporti Europei, 2016(61): 73207.

[14] Jung, J., Chen, L., Sohn, G., Luo, C., Won, J.U. (2016). Multi-range conditional random field for classifying railway electrification system objects using mobile laser scanning data. Remote Sensing, 8(12): 1008. https://doi.org/10.3390/rs8121008

[15] Corman, F., Meng, L. (2014). A review of online dynamic models and algorithms for railway traffic management. IEEE Transactions on Intelligent Transportation Systems, 16(3): 1274-1284. https://doi.org/10.1109/TITS.2014.2358392

[16] Riejos, F.A.O., Barrena, E., Ortiz, J.D.C., Laporte, G. (2016). Analyzing the theoretical capacity of railway networks with a radial-backbone topology. Transportation Research Part A: Policy and Practice, 84: 83-92. https://doi.org/10.1016/j.tra.2015.03.018

[17] Kiesel, F., Ries, J.M., Tielmann, A. (2017). The impact of mergers and acquisitions on shareholders' wealth in the logistics service industry. International Journal of Production Economics, 193: 781-797. https://doi.org/10.1016/j.ijpe.2017.09.006

[18] Binder, S., Maknoon, Y., Bierlaire, M. (2017). The multi-objective railway timetable rescheduling problem. Transportation Research Part C: Emerging Technologies, 78: 78-94. https://doi.org/10.1016/j.trc.2017.02.001

[19] Kumru, M., Kumru, P.Y. (2014). Analytic hierarchy process application in selecting the mode of transport for a logistics company. Journal of Advanced Transportation, 48(8): 974-999. https://doi.org/10.1002/atr.1240

[20] Sharp, C., Woodcock, J., Sica, G., Peris, E., Moorhouse, A.T., Waddington, D.C. (2014). Exposure-response relationships for annoyance due to freight and passenger railway vibration exposure in residential environments. The Journal of the Acoustical Society of America, 135(1) 205-212. https://doi.org/10.1121/1.4836115

[21] Lee, Y.J., Kim, R.E., Suh, W., Park, K. (2017). Probabilistic fatigue life updating for railway bridges based on local inspection and repair. Sensors, 17(4): 936. https://doi.org/10.3390/s17040936

[22] Mohamed, A., Peng, Q., Abid, M.M. (2019). The measurement of high-speed railway (HSR)-precision engineering. Civil Engineering Journal, 28(4): 517-522. https://doi.org/10.14311/CEJ.2019.04.0042

[23] Ha, O., Choi, B.L., Kim, Y., Kim, B., Lee, K.D. (2015). Development of indicators of freight stations for digital convergence. Cluster Computing, 18(1): 269-278. https://doi.org/10.1007/s10586-014-0386-8

[24] Nourbakhsh, S.M., Bai, Y., Maia, G.D., Ouyang, Y., Rodriguez, L. (2016). Grain supply chain network design and logistics planning for reducing post-harvest loss. Biosystems Engineering, 151: 105-115. https://doi.org/10.1016/j.biosystemseng.2016.08.011 